\title{
Clinical and non-clinical healthcare workers faced similar risk of acquiring 2009 pandemic H1N1 infection
}

\author{
PTY Ching ${ }^{1 *}$, CHS Lam ${ }^{1}$ BJ Cowling ${ }^{2}$, WH Seto ${ }^{1}$ \\ From International Conference on Prevention \& Infection Control (ICPIC 2011) \\ Geneva, Switzerland. 29 June - 2 July 2011
}

\section{Introduction / objectives}

In the $2009 \mathrm{H} 1 \mathrm{~N} 1$ pandemic, the Hospital Authority managing $>90 \%$ of hospital beds and 74 outpatient clinics in Hong Kong implemented mandatory reporting for healthcare workers (HCWs) with confirmed pH1N1 and collected detailed data on infected HCWs.

\section{Methods}

Under mandatory reporting, HCWs with influenza-like illness must present themselves to staff clinic and tested for pH1N1 by RT-PCR and viral culture. A confirmed case was defined as positive on either test. A standard questionnaire was used to assess clinical presentation and nature of exposure. Clinical staff were defined as HCWs involved in direct patient care and non-clinical staff are those without. The reporting for all staff began on 17 June until 31 August 2009. From 1 September 2009 , it was mandatory only for clinical staff until 31 May 2010 when the pandemic was downgraded Infection control guidelines were issued on 29 April 2009 and education sessions were attended by $>39,000$ staff.

\section{Results}

During staff mandatory reporting, there were 249 confirmed pH1N1 cases among 40,511 clinical staff $(0.62 \%)$ and 119 among 18,759 non-clinical staff $(0.63 \%$; $\mathrm{p}=0.82)$. The relative risk for clinical versus non-clinical staff was 0.98 (95\% CI, 0.78-1.20). In the entire reporting period, a total of 1039 (2.6\%) clinical staff had pH1N1 infection. Among clinical staff, 212/1039 (20\%) cases reported contact with a confirmed pH1N1 infection, similar to the $24 / 119$ (20\%) from non-clinical staff. Importantly, unprotected exposure to a colleague confirmed with pH1N1 were 10-fold higher than exposure to infected patients, similar for clinical (9\%) and non-clinical (8.4\%) staff $(\mathrm{p}=0.97)$.

\section{Conclusion}

Attack rate was similar for clinical and non-clinical staff showing no increased risk in clinical care.

\section{Disclosure of interest}

P. Ching: None declared, C. Lam: None declared, B. Cowling Grant/Research support from MedImmune Inc., W. H. Seto Other Presented in anti infective meeting 2010 by Pfizer.

\section{Author details}

'Hospital Authority, School of Public Health, The University of Hong Kong, Hong Kong, China. ${ }^{2}$ Infectious Disease Epidemiology Group, School of Public Health, The University of Hong Kong, Hong Kong, China.

Published: 29 June 2011 